\title{
Law of Physics 20th-Century Scientists Overlooked (Part 7): Steady State Cosmic Structure
}

\author{
Conrad Ranzan \\ Cosmology Department, DSSU Research, Niagara Falls, Canada \\ Email: Ranzan@CellularUniverse.org
}

How to cite this paper: Ranzan, C. (2021) Law of Physics 20th-Century Scientists Overlooked (Part 7): Steady State Cosmic Structure. Open Access Library Journal, 8: e7901. https://doi.org/10.4236/oalib.1107901

Received: August 29, 2021

Accepted: December 14, 2021

Published: December 17, 2021

Copyright $\odot 2021$ by author(s) and Open Access Library Inc.

This work is licensed under the Creative Commons Attribution International License (CC BY 4.0).

http://creativecommons.org/licenses/by/4.0/

(c) (i) Open Access

\begin{abstract}
Presented is an exploration into the operation of the Universe that begins with a single assumption, an empirical proposition accepted by virtually all theorists. The article then builds on this assumption (a fundamental process of expansion) by applying and integrating three important laws of physics that $20^{\text {th }}$-century scientists overlooked-namely those detailed in Parts 1,2 , and 4 in this series of articles. They are, respectively, 1) "The velocity differential propagation of light," which presents the definitive cause of the cosmic redshift. 2) "Energy generation via velocity differential blueshift," which details Nature's fundamental energy amplification process. And 3) "Mass extinction by aether deprivation process," which gives the extraordinary explanation of how Nature annihilates mass; but not in the sense of ordinary destruction, rather, this is annihilation in the irreversible terminal sense. The assembled system is then used to make a number of model-specific predictions. The theory-anticipated patterns and the actually observed evidence are compared and shown to be in remarkable agreement. The profound conclusion is that the patterns of the Universe's cosmic-scale structure are not phenomenological but are inherent. Cosmic structure exists by virtue of a perpetual self-sustained mechanism-a timeless steady state system.
\end{abstract}

\section{Subject Areas}

Cosmology, Astrophysics, Fundamental Physics

\section{Keywords}

Cosmology, Steady State Systems, End-State Neutron Stars, Blueshift Process, Mass Extinction, Galaxy Patterns, Cluster Periodicity, Cosmic Cellular Structure, Aether, DSSU Theory 
Indeed, the process of scientific discovery does not consist of a search for axioms but a search for empirical propositions from which new axioms may be induced; at any stage in the development of a science the axioms are temporary structures that must be added to as the science grows.

-J. Bronowski, Scientific American, June 1964

\section{The Determiners of Cosmic-Scale Structure}

\subsection{Expansion, the Empirical Proposition}

The most fundamental determiner of cosmic structure is the expansion of the space medium. As Leonard Susskind, an accredited expert on the cosmic landscape, has stated, regarding the expansion, the growth, of the space medium; it seems rock solid, no cosmologist questions it [1]. Whatever one chooses to call it - the vacuum, the quantum foam, the cosmic fabric, or the aether-it is the emergence/expansion of such medium that sustains vast near-empty regions. It is the emergence of new "space" or new vacuum-what herein will be called aether-that sustains the existence of cosmic Voids. (The specific nature of the aether will be explained later.)

The existence of voids is a fact based on observational evidence. However, the mechanism underlying their existence is, at this stage of the discussion, an assumption. No, not an ordinary assumption; rather, because of the obvious evidence, "expansion" should be elevated to an empirical proposition. The plan is to see where this leads. Based on the proposition that the universal space medium, aether, has the innate ability to expand, one should expect a "landscape" of Voids.

Notice that the "expansion" assumption was prudently limited; the assumption was not extrapolated to whole-universe expansion. The claim is not that aether emergence/growth sustains the expansion of the Universe. The definitive reason for refraining from this sort of speculation will be explained in a moment.

First, recognize that there is a serious potential problem with having any pattern of Voids as presented in Figure 1. It is clearly evident that all the galaxies, stars, dust and gas - all the material stuff of the universe - tend to aggregate within the pockets between the Voids. Over time, the pockets of aggregations, the galaxy clusters, in the absence of some countering mechanism, would undergo gravitational collapse. Ultimately, everything would vanish into an end-state object, what is popularly called a black hole.

Having everything vanish into blackness certainly makes for a bleak future; but that's not the real problem. Total gravitational collapse is plausible and readily understandable [2]. No, the deeper problem is the question where all the matter came from in the first place. Where indeed? The implication is that the universe must have had a beginning! But such a philosophically-unsound concept only makes the problem worse, only deepens the bafflement. Yet that is exactly the course the scientist of the $20^{\text {th }}$ century followed. They adopted and 


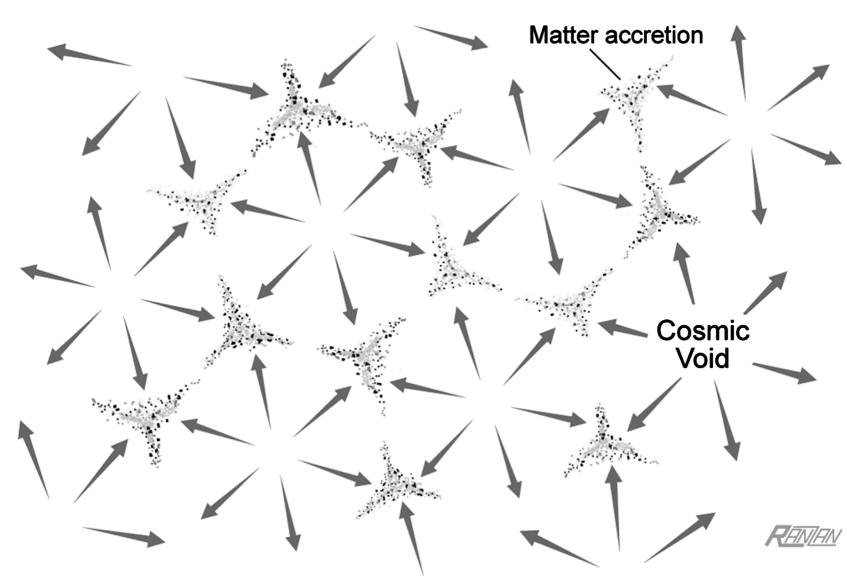

Figure 1. Cosmic Voids are sustained by aether emergence/expansion as shown in the schematic cross-section view. Whatever mass there is in such a universe "landscape" must logically accrete at the interfaces between the voids.

embraced the view of universe genesis, a mathematically-derived grand event, imagined as a real event despite lacking a proper physics foundation. The result? The legacy has not been one of enlightenment. Rather, what have been passed on to the $21^{\text {st }}$ century are new and embarrassing levels of bafflement as to how the universe operates. The $21^{\text {st }}$ century inherited a problem-plagued cosmology.

And it all began with the adaptation of a faulty assumption-an unscientific extrapolation of misinterpreted redshift evidence.

\subsection{Steady State by Default}

The assumption that the space medium, aether, expands is a good one. The evidence supports it.

The assumption that the universe expands is wholly misguided. There is absolutely no evidence. There is no Hubble expansion; no systematic recession of distant galaxies, nor distant clusters. Scientists had simply overlooked the true cause of the cosmic redshift-the true cause being the velocity differential redshift mechanism [3]. (The velocity differential refers to the motion of the aether as it affects the wavelength of the propagating light waves.) The proof was presented as Part 1 in the "overlooked laws" series of articles, and was first proposed in 2014 in the published article Cosmic Redshift in the Nonexpanding Cellular Universe ... [4]. The proof is impeccable.

In addition to the lack of evidence, the assumption of uninhibited expansion has a troubling philosophical aspect. Given that the Universe is infinite, how can it expand to become more infinite?! Given that the Universe is infinite, why would it bother to become more infinite?!

Needless to say, there are numerous other problems, a discussion of which would take too long and divert attention from the main point. But do be aware that the Big Bang experts (like physicist Sean M. Carroll) readily admit, their expansion and accelerating scenario leads to a Preposterous universe.

Also, as mentioned above, there is the unresolvable problem of the origin of 
the Universe-some unimaginable all-encompassing beginning that issues from a theoretical backward-in-time extrapolation. The problem is as serious as it could possibly be: a complete breakdown of physics.

Thus, by not making this kind of an unscientific assumption (not to mention, philosophically untenable) one is left with the default position. This is a position that requires no assumption about the Universe, other than asserting that The Universe IS. Period. This is the default position.

The default position is that the universe, the real Cosmos, is a steady state Universe.

By rejecting the "Preposterous" assumption of whole-world expansion and simply recognizing the universe's steady-state status, it stands to reason that just as the Voids always remain as they are, so too, the galaxy clusters remain coherently in their proper places. While Voids are sustained by the expansion process; galaxy clusters have their own sustaining mechanism-a mechanism of ongoing matter regeneration.

\subsection{Matter Regeneration}

It is here, with the mechanism for the regeneration of mass and energy particles, that several overlooked laws of physics function as essential components:

- The Blueshift process. It affects photons and neutrinos and occurs within the surface of end-state neutron stars [5].

- The escape mechanism. This is the means by which photons and neutrinos are able to break free of what is otherwise an impassible barrier enveloping each end-state neutron star [6].

- The aether deprivation process. It is a process that is self-explanatory, once you understand that the existence of all matter is dependent, utterly and completely, on the presence of aether [7].

- The transformation of the escaped radiation (the photons and neutrinos emitted by the end-state neutron stars) into mass particles. This, of course, is a standard physics phenomenon. It represents the last step of the matter regeneration mechanism.

One more item is needed. What has not yet been mentioned is gravitation. Since it is the most important force-like effect governing the universe, its inclusion here is unavoidable. The question is Which gravity? ... Categorically, there are only 3 models to choose from: the force type (Newtonian gravity), the geometric class (Einstein's curved continuum), and the aether theory of gravity (DSSU nonmaterial aether). Since the DSSU ${ }^{1}$ aether theory of gravity is the only known model that unifies all five of gravity's aspects and agrees with observations [8], it is the one that will serve as the backdrop for the following detailed explanation of the matter regeneration mechanism.

The detailed workings of DSSU aether gravity are not needed here. For the most part, one only needs to remember this critically important aspect of aether gravity theory: The simple fact is that all particles absorb/consume aether, and ${ }^{1}$ DSSU is the acronym for the Dynamic Steady State Universe. 
thereby sustain a continuous inflow of more aether.

\subsection{Matter Regeneration, the Blueshift Process}

The Blueshift process takes place within the surface of end-state neutron stars.

The end-state neutron star, also known by the shorter term Terminal star, is the most extreme object (as a contiguous entity) found in the universe.

Because it contains mass and energy, it absorbs and consumes aether (as a fundamental aspect of the aether theory of gravity).

Because it is the most extreme type of contiguous body, the inflow of aether at its surface is equal to the speed of light $c$.

Because the Terminal star's surface is a pure energy surface, the aether inflowing at lightspeed there (at the surface) is NOT a violation of special relativity. It is self-evident. As shown in Figure 2, the energy surface consists of photons and neutrinos all "propagating" radially outward, while aether is flowing radially inward. The speeds are equal; the directions are opposite. Thus, these energy particles remain "stationary." They remain trapped within the Terminal star's energy layer. [5]

Why only photons and neutrinos, and not other particles? It is simply because they are the only particles known to travel at the speed of light. Only they can propagate in-place against the lightspeed inflowing aether.

The remarkable thing is that while trapped in the energy layer the photons and neutrinos gain in energy. In other words, while propagating in the surface layer, these particles undergo a gradual, but relentless, wavelength contraction. The proof of this is surprisingly simple.

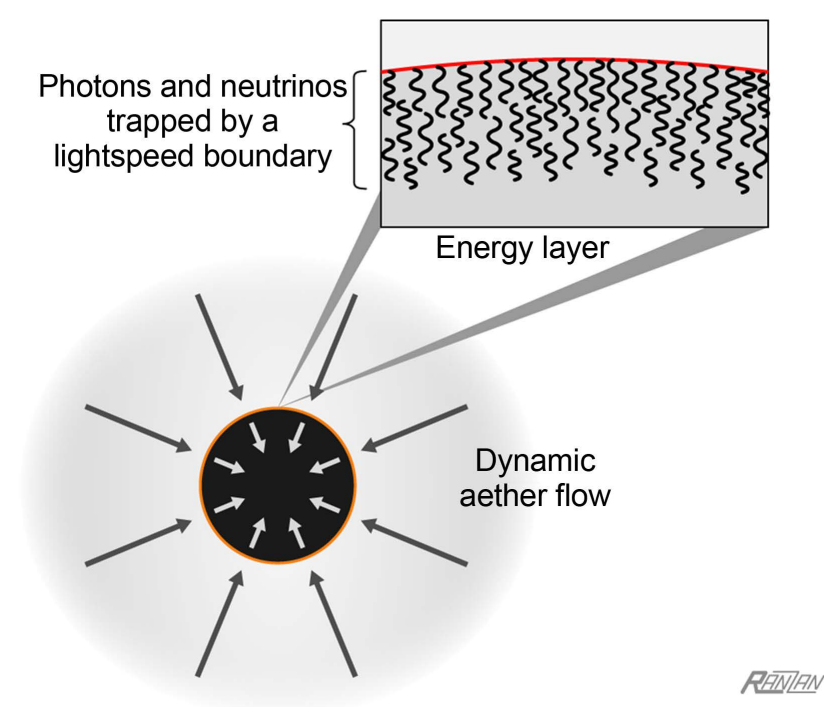

Figure 2. Cross-section view of a Terminal star (an end-state ultra-dense mass) revealing its unique surface layer. It is a thin zone consisting of photons and neutrinos. These particles are propagating outward; but because the aether is flowing inward with the same speed, the photons and neutrinos simply remain stationary within the energy layer. The crucial aspect is that the propagation is taking place within a blueshifting region-in accordance with the Principle of velocity differential propagation. 
Consider a representative photon trapped in the surface, as shown in Figure 3. In accordance with the aether theory of gravity, the magnitude of the inflow speed (of aether) varies as indicated by the graph. Obviously there exists an inflow velocity difference at the photon's location. The photon "experiences" a velocity difference between its two ends. When analyzed, it is found that the front and back ends are actually moving closer together [6].

Given that the photon always travels at speed $c$ with respect to the aether medium, the following must be true.

$($ Relative velocity between ends of photon $)=($ vel of front end $)-($ vel of back end),

$$
\begin{aligned}
& =\left(c+v_{1}\right)-\left(c+v_{2}\right), \\
& =c+v_{1}-c-v_{2}, \\
& =\left(v_{1}-v_{2}\right)<0 .
\end{aligned}
$$

Note that aether flow $v_{1}$ and $v_{2}$ are both negative. But because $v_{1}$ is more negative than $v_{2}$, the bracketed expression must be negative-indicating a converging situation.

Consequently, any surface-embedded photons and neutrinos undergo energy amplification - they slowly gain energy.

If nature is to harness this energy generating process, there needs to be a way for this recharged radiation to escape the Terminal-state stars.

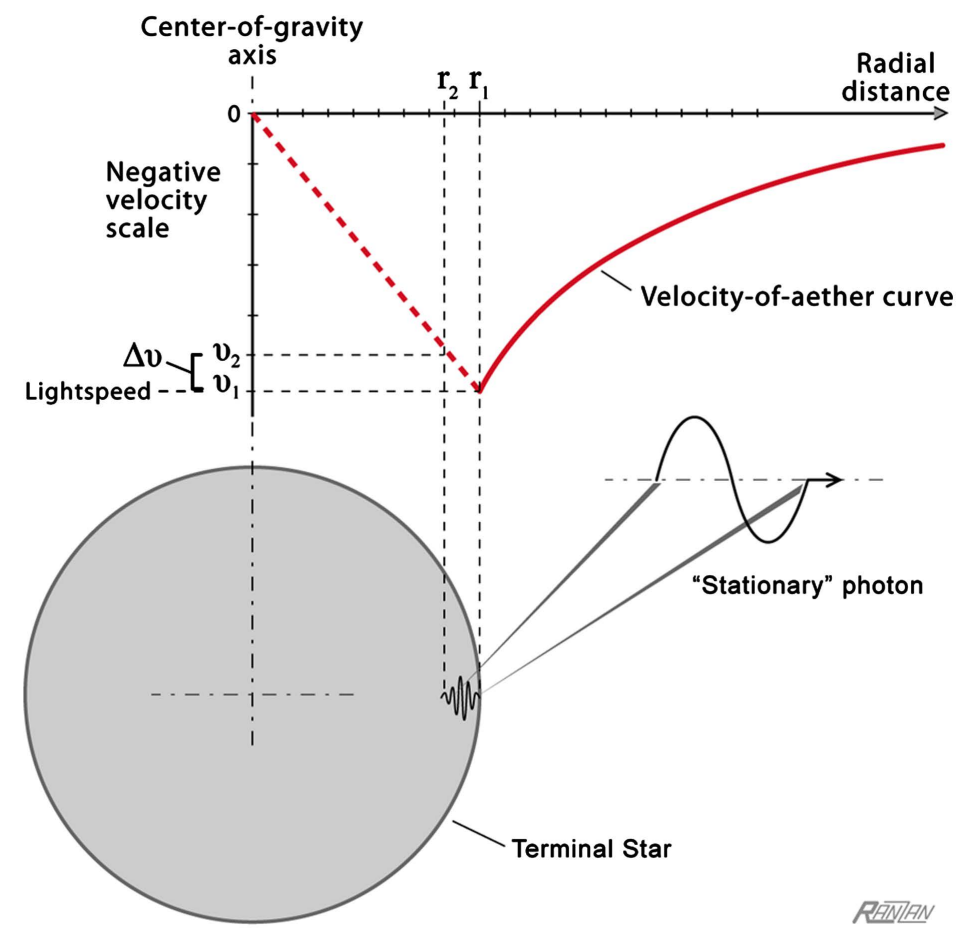

Figure 3. Surface embedded radiation gains energy via the Blueshifting process. The representative surface photon, by virtue of propagating "in place" within a zone of decelerating aether, is subjected to continuous wavelength contraction. (The front and back ends of the photon "experience" a flow differential $\Delta v$.) Essentially, it slowly gains energy. 


\subsection{Matter Regeneration, the Escape Mechanism}

Neutron stars, including Terminal stars, are known to possess powerful magnetic fields. Rapid rotation causes the lines of force of these magnetic fields to become collimated. And keep in mind that significant rotation is practically always present at some stage in the gravitational collapse (or gravitational aggregation) leading to the Terminal structure. Terminal stars may be pictured as having very tight magnetic beams (one at each pole) with truly enormous energy densities.

Now remember what was said about mass and energy? They both require a flow of aether to sustain their existence. It follows that when there is a greater mass density, more aether must flow into the same volume; when there is a greater energy density, more aether must be consumed. The collimated magnetic field represents an extraordinarily high energy density and is, therefore, a prodigious consumer of aether. And this voracious extraction of aether is the key - it precludes the formation of a lightspeed boundary at the poles, at the bases of the tightly-bundled magnetic force lines (Figure 4).

In other words, a Terminal star's magnetic field provides the channels through which those surface photons and neutrinos are able to escape. Once they reach

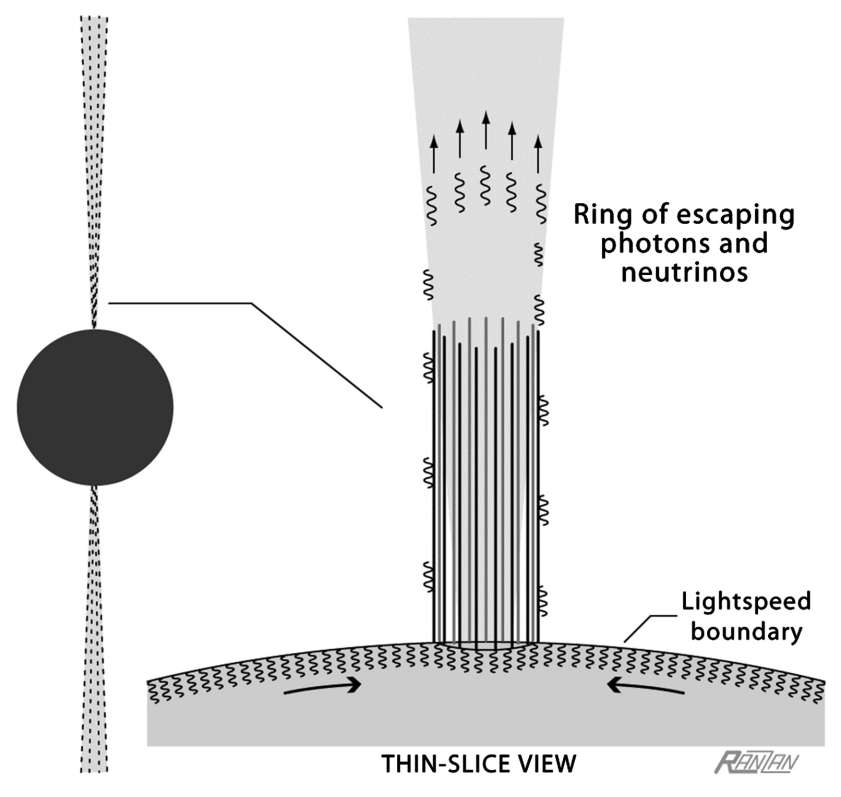

(a)

(b)

Figure 4. Escape mechanism of photons and neutrinos. Radiation escapes from the Terminal star (a) by means of the opening in the lightspeed boundary (b). The openings originate with the neutron star's magnetic field and are maintained in the "open" state by the collimated nature of the magnetic field and the intense particle beam itself, both of which are voracious absorbers of aether. Both retard the radial inflow of aether. The lateral pressure, due to the extreme density of the energy surface, drives the radiation particles toward the edge of the portals from which they emerge as a ring of radiation. The streaming of escaping energy is a continuous phenomenon-sustained by the ongoing energy-generating Blueshifting process. (Note: The schematic does not show the helical twisting caused by structure rotation, which is almost always present.) 
the opening, they escape. They shoot out at lightspeed; but note, this speed is not with respect to the surface but, rather, with respect to the inflowing aether.

A more detailed description is presented in the article, published in 2018, Natural Mechanism for the Generation and Emission of Extreme Energy Particles [6].

It should be emphasized that this energy dispersal mechanism operates continuously. There is no theoretical limit to the amount of energy that Terminal stars can expel into the greater cosmos. Remember, Terminal stars cannot collapse further, they can never become smaller, and they can never become bigger (as will be explained in Section 1.7)—regardless of mass input.

Next is an examination of what happens to this limitless energy flow.

\subsection{Matter Regeneration, the Formation of Mass Particles}

Under this topic covering mass formation/creation it is import to realize that Terminal stars produce and eject the most energetic particles to exist in the universe. No other process even comes close-no collision, no other stellar phenomenon, and no manmade particle accelerator can approach the extremes generated by Terminal stars. For example, neutrinos having energy in the peta-electron-volt range (that is, $10^{15} \mathrm{eV}$ ) have been detected by the IceCube Neutrino Observatory located at the South Pole [9]. The journal New Scientist (2016, April 30-May 6) reported "Neutrinos captured at the South Pole carry more energy than we [the experts] can explain." To give another example, this one from 2017 September: "The neutrino that triggered everything last September in IceCube was at approximately 300 tera electron volts $\left(3 \times 10^{14} \mathrm{eV}\right)$-nearly a factor of 300 million times more energetic than the neutrinos that come prolifically from the fusion production in our sun." So said Darren Grant, an Associate Professor of Physics at the University of Alberta and a spokesperson at the IceCube Observatory.

Terminal stars are also responsible for the emission of ultra-energetic photons; such ejecta are observable and recognized by astronomers as gamma-ray bursts.

It is entirely within the realm of standard physics that gamma and ultra-gamma photons can generate mass particles. To borrow a line from physicist Frank Wilczek, "You start with massless particles and you get mass." The ability to create matter from light is amongst the most striking predictions of quantum electrodynamics. Experimental signatures of this have been reported in the scattering of ultra-relativistic electron beams with laser beams, intense laser-plasma interactions and laser-driven solid target scattering [10].

Turning to neutrinos, the mass generating ability of some neutrons can be truly astonishing. A neutrino in the $\mathrm{PeV}$ category has the equivalent mass energy of about one million resting protons. This means a PeV neutrino has the potential to "create" a million protons. And not to be overlooked is the fact that the neutrino will, sooner or later, strike some preexisting mass and transfer its energy-a large portion of which will go into the formation of new mass particles. 
Typically, expelled particles propagate through the universe until, sooner or later, near or far, they collide and interact with whatever lies in their path. The result (provided the incident particle has sufficient energy) is the production of new mass particles. Naturally, this mass regeneration/formation occurs predominately where matter is already present. The reasonable expectation is that more mass is generated in galaxies and galaxy clusters than in less dense regions.

\subsection{Matter Gain Is Balanced by Matter Loss}

Now pause and reflect on the picture developed up to this point. The potential problem of all galaxy clusters collapsing-of each cluster collapsing into some sort of single compact structure-has been solved, solved by the matter regeneration mechanism described above. But there is a new problem. This formation/creation process is perpetual-it literally has no limit. No quantity limit, no time duration limit. It is at this point that the mass-loss process enters the picture.

The process is called mass extinction by aether deprivation, and is remarkably easy to explain.

Recall, once again, the ontological fact about mass and energy: They require a continuous flow of aether to sustain their existence. It follows that when, for whatever reason, the available flow is insufficient, the vital flow is somehow restricted, then the unavoidable result will be the vanishment of the so-deprived mass.

Follow the simple logic:

- The greater the quantity of mass in a structure, the more aether must flow into that structure. It's straightforward. The more massive the star is, the more aether it needs to consume.

- Consider a spherical body. The demand for aether depends directly on the sphere's volume.

- The availability of aether, however, depends directly on the sphere's area. (The aether has to flow through the limited surface area; there is no other way to reach the mass.)

- Now since the volume is proportional to the cube of the radius, while the area is proportional to the square of the radius, the demand for aether can exceed the availability.

And this is exactly what happens when too much mass comes together. When that happens, aether deprivation will occur. It will occur at the core of the structure (Figure 5) [7].

Mass extinction by this process is a common occurrence. It generally happens when a multiple solar-mass star (or the star's core) suddenly changes in density, namely, when it collapses to the neutron-density state. Whenever a structure, or a mass aggregation, collapses and the result is a Terminal star, there will occur a significant loss of mass... Additional details of the collapse mechanism may found in reference [7]. 


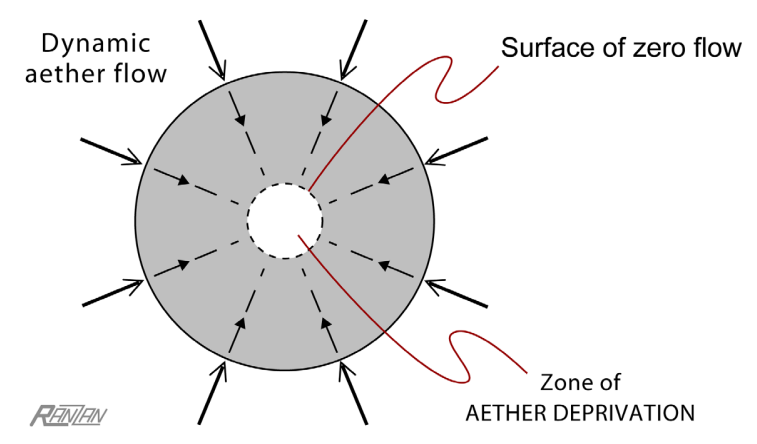

Figure 5. Mass extinction by the process of aether deprivation. The quantity of mass represented in the cross-section schematic exceeds the amount sustainable by the aether able to flow through the surface. Shown is the situation of aether being totally consumed by the time it reaches the "surface of zero flow." The result is a core region of aether deprivation - a place where mass instantly vanishes. What remains is a nothingness hole in the universe that instantly closes. This sequence, considerably simplified here, generally occurs when a multiple solar-mass star (or star core) suddenly changes in density, viz, when it collapses to the neutron-density state.

As a point of interest, Terminal stars are found wherever normal stars exist. But they are most abundant within the supermassive cores of galaxies.

A final point on the resolution of the mass problem. It has just been shown that mass formation (brought about by energy generation) is effectively countered by mass extinction. But in this resolution, we find something deeper, something profound. Terminal stars, it turns out, are creators AND destroyers. They are the energy generators for mass formation as well as the uncompromising destroyers of mass. They are the key to the maintenance of the universe's material flow as a steady state system.

With all the determiners of cosmic structure in place, it becomes possible to predict the distribution of matter, to predict the very shape of cosmic structure. And if everything described so far is rooted in reality, if those "overlooked" factors and processes are valid determiners, then their combination as a coherent system should lead to meaningful predictions-various features and phenomena that should be observable.

\section{Prediction of a Natural Cosmic Pattern}

A conviction of philosophers across the ages and of contemporary scientists has been that knowledge of the proper laws of physics would enable mankind to predict the universe as it is known to be, and to elucidate its key features.

And so it is. The universe is known to be cellular; and now, with the proper laws of physics in place, what is known and what emerges from prediction can be compared.

The determiners of cosmic structure, when conceptually combined, present a picture with two dominant effects:

- Contractile gravity of the steady state galaxy clusters;

- Expansion effect of the steady state Voids. 
Then, when these effects are combined into a 2-dimensional pattern, they predict a hexagonal pattern as in Figure 1.

Turning to the full-dimensional real world: When the Voids as fonts of aether are combined with galaxy clusters as the sinks of aether-combined into a 3dimensional pattern-the prediction is a close-packed array of rhombic dodecahedra. For the proof, see the article Large-Scale Cell Structure of the Dynamic Steady State Universe [11]. An isolated cell (schematic) is shown in part (a) of Figure 6; and a representative close-packed array of such cells is presented in part (b). Notice that the prediction of the rhombic dodecahedral structure automatically includes the prediction of two distinct cluster sizes. The smaller class is here associated with the Minor nodes and the larger class is associated with the Major nodes. Thus, there are 8 "small" clusters and 6 "large" clusters surrounding each Void.

The rhombic dodecahedron is a polyhedron with a most unusual shape, as can be seen in Figure 7. Depending on the orientation, its profile can appear as a hexagon or as a square. The unusualness of the polyhedral shape is responsible for some remarkable patterns that are revealed in an extended dense packing.

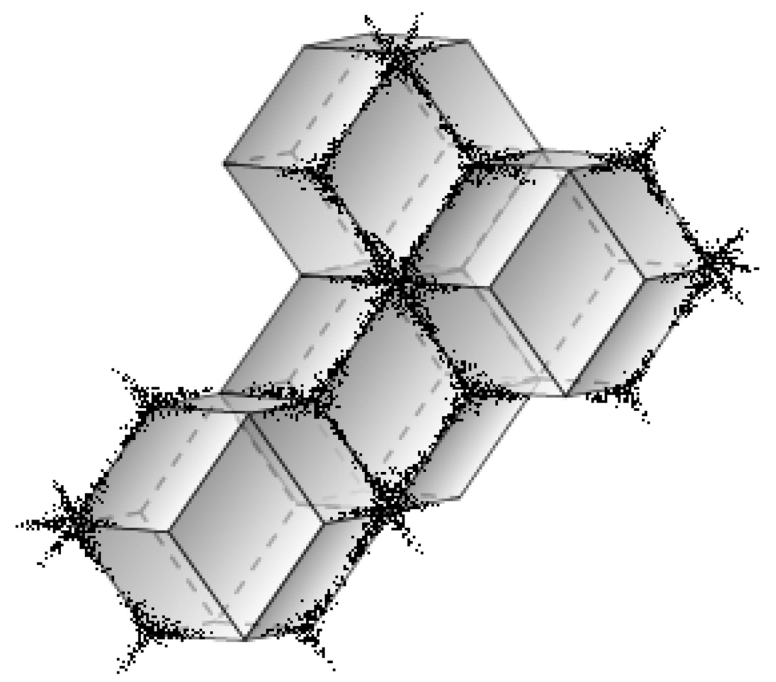

(b)

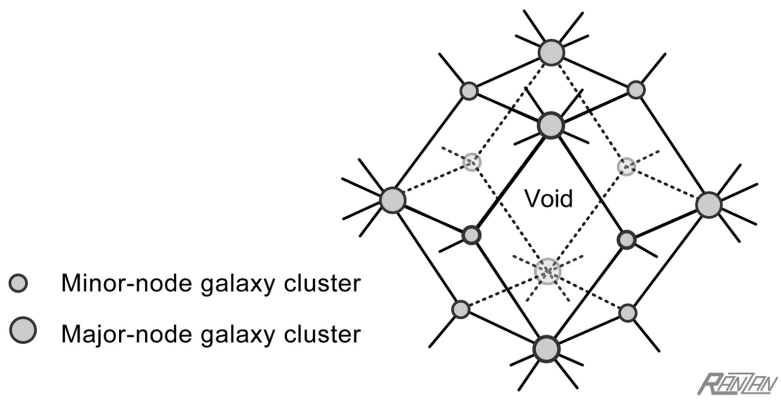

(a)

Figure 6. Predicted cosmic structure. Rhombic dodecahedral cosmic cell (a). Schematic of a three-dimensional grouping - a close-packed array of rhombic dodecahedra (b). 


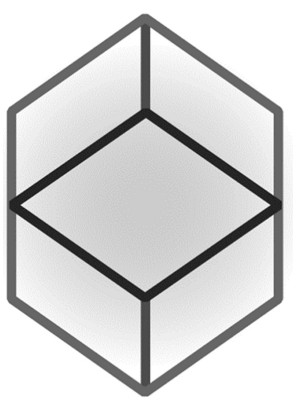

Face-on view

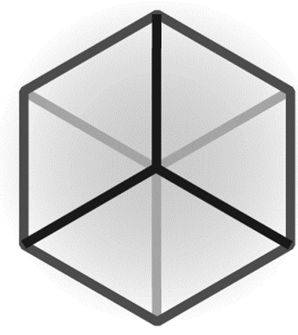

Looking down on Minor node

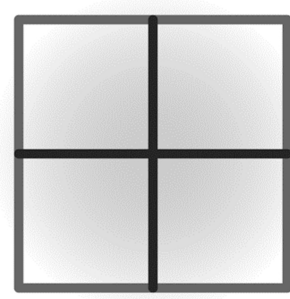

Looking down on Major node

Figure 7. Stick model of a rhombic dodecahedron viewed along three different axes of symmetry reveals two hexagonal profiles and an unexpected perfect square.

Familiarity with this shape will be quite useful for understanding and interpreting the evidence presented in the next section.

The cosmic-scale cellular structure, of the void-cluster network, being predicted is radically different from the old view. Under the $20^{\text {th }}$-century view cellular structure was merely a phenomenological condition, while under the $21^{\text {st }}$ century view-the DSSU world view-it is an intrinsic state.

\section{Structure Prediction Matches Observational Evidence}

Kant thought deeply about the relation between theory and observation. He recognized that a scientific cosmology must be based on observation, but he also recognized a less obvious and less widely appreciated truth - that only observation guided by theoretical insight is likely to uncover the deep regularities underlying phenomena.

-David Layzer, Constructing the Universe (p. 114)

First, the obvious evidence: The Universe is cellularly structured. The evidence is abundant and incontrovertible.

For many decades, beginning in the 1970s, Estonian astronomer Jaan Einasto reported on galaxy surveys that revealed astonishing structures. Galaxies were distributed in the form of long filaments and giant walls. He spoke of the distribution of galaxies being a honeycomb with huge voids which contained practically no galaxies.

By the 1980s astronomers were routinely reporting that real superclusters consist of multiple intertwined filaments. And they never failed to mention the great voids.

The general consensus is that the largest structures in the universe are membrane-like distributions of galaxies enclosing enormous voids, and as physicist Evan Harris Walker pictures it, "as if the galaxies were the molecules in the foam on some giant mug of beer."

On the basis of overwhelming evidence, cosmologists now recognize the architecture of the Universe as being a vast interconnected system of filamentary structures and great voids-a Veronoi honeycomb ([12], p. 67). 
Galaxy clusters (the nodal structures) come in two sizes. Just as was predicted with the close-packed dodecahedron (Figure 6), clusters fall into two categorical sizes. Back in 2002, following a study of 79 distant clusters of galaxies (redshift range $0.1<\mathrm{z}<1$ ), astronomers Naomi Ota and Kazuhisa Mitsuda announced the "discovery of two classes of cluster Size." They presented a histogram, based on the measured core size of each cluster, revealing a distinct doublepeaked distribution. The pattern cannot be explained by any selection bias or instrument effects; they, therefore, concluded that it reflects the real nature of the clusters.

The evidence was clear "the histogram of the core radius shows two peaks at 60 and $220 \mathrm{kpc."} \mathrm{Cluster} \mathrm{core} \mathrm{diameters} \mathrm{could} \mathrm{be} \mathrm{classed} \mathrm{as} \mathrm{either} \mathrm{391,000} \mathrm{ligh-}$ tyears or 717,000 lightyears. However, Naomi Ota and Kazuhisa Mitsuda were baffled by the underlying cause. They failed to apprehend "through which physical processes such discrete cluster structures are formed.” [13]

Supergiant elliptical galaxies. One of the predictions (but not mentioned earlier) is that there will always be at least one supergiant "elliptical" galaxy at each node of the dodecahedral structure. This means that such a galaxy should always be present at the center of a nodal galaxy cluster.

Astronomers refer to these giants as $c D$ galaxies $^{2}$ and have long confirmed the prediction. At the center of any significant cluster there is always to be found a giant dominant galaxy. Importantly, they are nonrotating or have negligible rotation and "tend to outshine the next brightest cluster galaxies by as much as a factor of two." [14] The best known examples include M87 at the center of the Virgo cluster (our nearest nodal cluster), ESO444-46 in the Shapley Cluster (aka A3558 cluster), and NGC4874 at the heart of the Coma Cluster.

Right-angled walls of galaxies. The eminent astronomer Anthony P. Fairall (1943-2008) repeatedly reported on finding walls of galaxies having right-angled bends. He and his colleagues emphasized the usefulness of such a feature as a critical test for any theoretical model. They had immediately realized that if the 90-degree bends are real, as it was believed they are, then any theory based on randomness would be untenable.

But the right-angle feature is precisely what the dodecahedral shape predicts, as is clearly evident in the right-hand image of Figure 7. Theory agrees with observation.

In 2000, a Mexican professor of astronomy, Renée C. Kraan-Korteweg, published a study focusing on the galaxies behind the Milky Way in the direction of the Great Attractor [15]. The professor pointed out that only in recent years have astronomers developed the techniques to peer through the Milky Way's dense disk, the gas-and-dust region called the Zone of Avoidance, and uncover the formerly hidden distribution of galaxies.

One of the startling discoveries was an unmistakable right-angled wall of galaxies directly behind the Zone of Avoidance (between Galactic longitude 200

${ }^{2}$ The " $\mathrm{c}$ " stands for supergiant, the "D" stands for diffuse outer envelope. 
and $\left.350^{\circ}\right)$. But lacking a theory or any kind of reasonable explanation for ninety-degree bends, the professor chose to describe the pattern of galaxies as a thin wave-a "continuity of ... thin filamentary sine-wave-like structure that dominates the whole southern sky and crosses the Galactic Equator twice." [15] The structure is, by cosmic standards, relatively near to us, as evident by its quite large angular measurements, being $\Delta 150^{\circ}$ (longitude) by $\Delta 75^{\circ}$ (latitude). The redshift distance, as Kraan-Korteweg's figure number 14 indicates, is $500<v<$ $3500 \mathrm{~km} / \mathrm{s}$, which places the structure at a distance of not more than 190 million lightyears.

Wall-like structures. So-called pencil beam redshift surveys have revealed remarkable regularity in the distribution of galaxies. A notable example is that of R. Broadhurst and colleagues, which involved a deep-space $(z<0.5)$ survey aligned with the polar axis of the Galaxy and extending out to distances of more than $1 \mathrm{Gpc}$ in two opposite directions. The data revealed a succession of walllike galaxy concentrations at fairly regular intervals-with an average spacing of about $130 \mathrm{Mpc}$. More than 13 such evenly-spaced "walls" of galaxies were found [16].

Motivated be these unexpected observations in the distribution of galaxies (redshift $\mathrm{z}<0.5$ ) a team of physicists undertook a statistical analysis "by comparing the data with models in which galaxies reside on the surfaces of bubbles or sheets."

The analysis indeed found that the structure was arranged into a regular cellular pattern. "It is striking that the standard deviation of the observed distribution is close to the most likely deviation from periodicity expected from a regular cellular structure." [17]

They found that the closest fit to the data was obtained with "a close-packed face-centered cubic lattice" of cosmic voids. Their statistical analysis revealed that cosmic voids are distributed ("packed together") in space according to the close-packed face-centered cubic arrangement. In terms of the density of the packing this is identical to the hexagonal closest packing of spheres [18] [19]which is exactly the configuration of the space-filling packing of rhombic dodecahedra. This is exactly what DSSU theory predicts!

It should be noted that the face-centered cubic close packing of spheres actually corresponds to a space-filling arrangement of a combination of rhombic dodecahedra AND rhombic-trapezoidal dodecahedra. This differs from the hexagonal closest packing only in that it reduces somewhat the symmetry of the cosmic tessellation.

The clearest explanation of wall-like structures (as well as their periodicity) can be found in a simple face-to-face assembly of rhombic dodecahedra. Figure 8 shows cells lined-up in a row so that they are all joined together via rhombic face to rhombic face. Be aware that an idealized rhombic dodecahedron has all its faces identical; thus, any face can be used, as long as the assembly follows a linear axis. The "walls" are most evident when the face-on row (a) is viewed from above-a view illustrated as row (b) in Figure 8. 


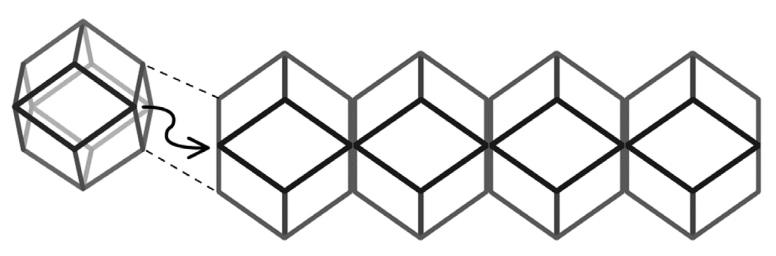

(a)

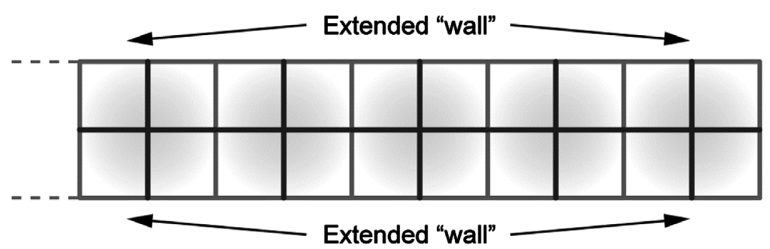

(b)

Figure 8. Wall-like structures can be explained with close-packed rhombic dodecahedra. Part (a) shows a row of such cells assembled so that rhombic face joins rhombic face; any face can be used, as long as the assembly remains linear. When viewed from above, as in Part (b), the "walls" are unmistakable as is their parallel nature. The latter aspect accounts for the reported periodicity

There is even evidence that the walls of galaxies are parallel. Fairall and his colleagues have stated, there is "the tendency for right-angled intersections and parallel structures to occur." "It is remarkable that this second wall [the Fornax Wall] runs virtually parallel $\left(<5^{\circ}\right)$ to the Sculptor Wall." [20]

Ribbon-like structure. Astronomers have reported the tendency of walls to be "ribbon-like" with filamentary "structures interconnect[ed] with one another to form the labyrinth." [21] The plane of the wall structure, in reference to the Cetus Wall, "appears to twist" and "to take on an almost ribbon-like characteristic." [22]

Look at the row of structures of Figure 8(a); note the predicted arrangement. Is there any better way to describe the string of rhombuses shown there as "ribbon-like"? Or as a "twisted" structure? The match between observation and prediction is impressive; the conclusion is clear. The Universe is a zig-zag labyrinth of galaxy distributions.

Great walls of galaxies. Great walls are said to be the largest structures of the Universe. The most famous is known as the Coma Wall (Figure 9), named after the Coma galaxy cluster at Right Ascension $13 \mathrm{~h}$ and first discovered by M. J. Geller and J. P. Huchra. Several others even longer have been found, such as the Sloan Great Wall. These structures are simply strings of Major nodes (the larger vertices of the dodecahedra) linked together by the zig-zag rhombic boundaries pictured in Figure 8(a).

Holes in the cosmic structure. The fact is, galaxies within wall structures are never uniformly distributed. Walls, as commonly observed, have gaps or holes. From another perspective, this is much the same as saying that the great voids are all interconnected [23]. As Anthony Fairall described it, "Were it [physically] possible, one could travel throughout the universe, passing from void to void." [21] The holes in great-wall structures were reportedly one tenth the size of the large voids separating the great walls themselves [20]. 


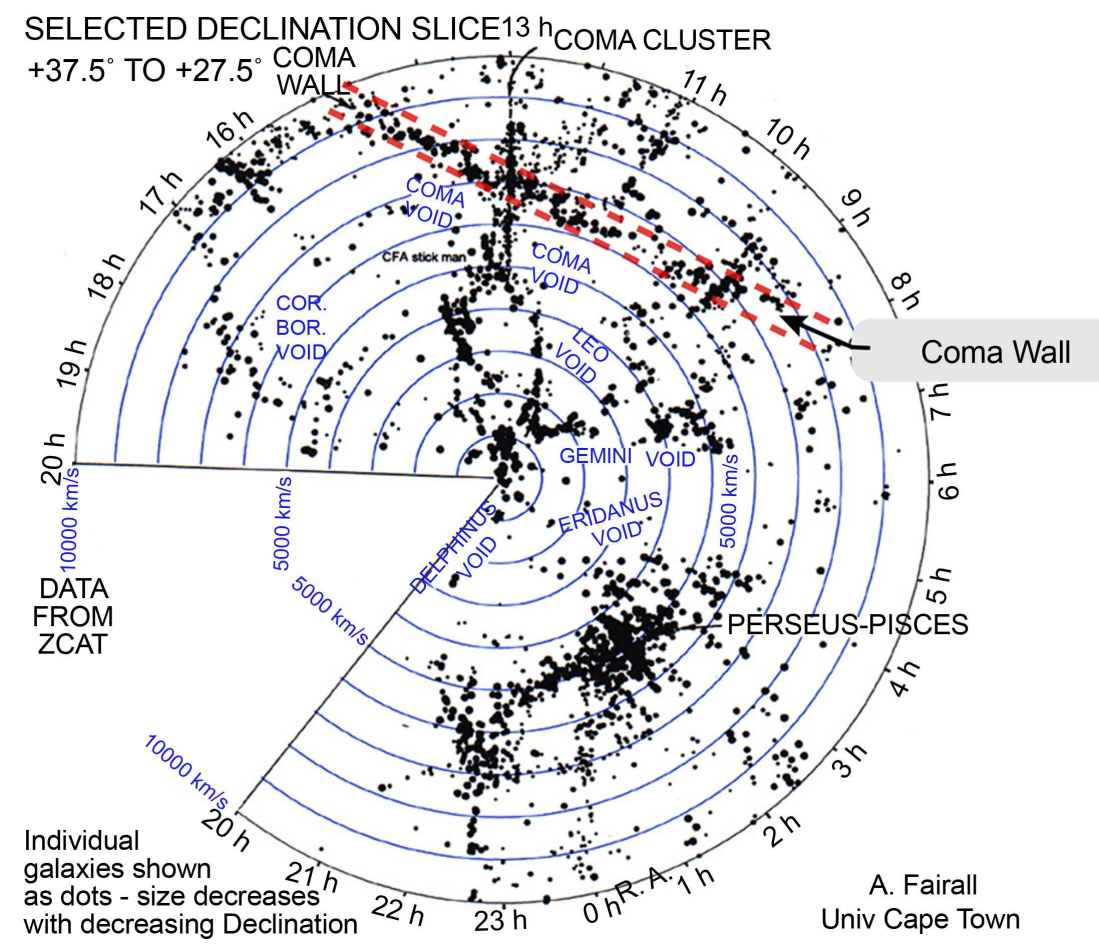

Figure 9. Best known example of an extended "wall" of galaxies is the Coma Wall (also known as the CfA2 Great Wall). When first discovered in the 1980s by M. Geller, J. Huchra, and V. Lapparent it was the largest known structure in the Universe. (Galaxymap image by permission of Estate of Anthony Fairall.)

Here, in a nutshell, is the theoretical explanation: The hole is simply the central portion, the least dense region, of a rhombic face or wall (Figure 10). The direction of motion (per aether gravity theory) is radially away from the rhombus center and towards the boundary edges and nodes-hence, the center is constantly being swept clean, so to speak. As for the size ratio mentioned, it is compatible with the predicted cellular structure.

Now, if one's line of sight passes through several such holes, something entirely possible since rhombic faces on opposite sides of a Void are more or less parallel to each other, it would seem as if one is looking into a deep cosmic cavity-a hole in the universe. Probably the most dramatic example of such a configuration is the "WMAP cold spot" located in the southern hemisphere of the celestial sphere in the direction of the constellation Eridanus. It was headlined in Scientific American (August 2016) as The Emptiest Place in Space and described as "A pocket of almost nothing [that] tells us something about the cosmos." The authors of the article also called it the "cold spot anomaly." The initial idea proposed by the experts was that the cold spot, this apparent hole in the universe, was some kind of a supervoid; but then more realistically suggested, "If several spherical voids are stacked next to one another in the direction of the cold spot (like a snowman), then the void could more easily explain its presence." That is, a snowman-like triple void could easily explain the presence of the observed cold spot. 


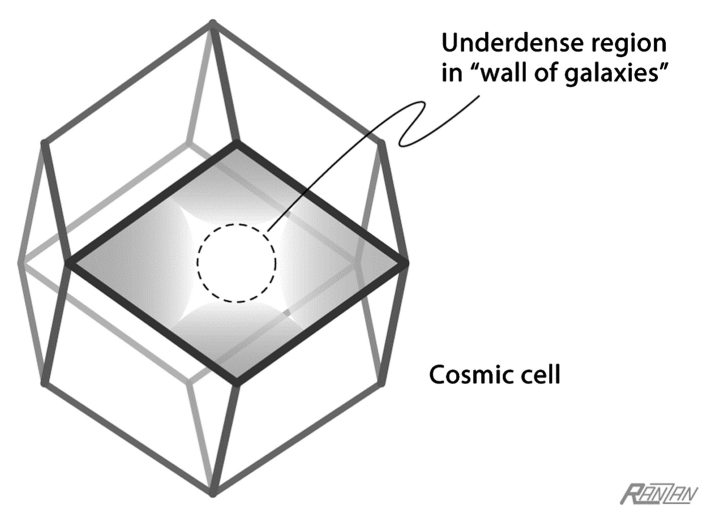

Figure 10. Schematic of a cosmic cell showing a rhombic-shaped portion of a "wall of galaxies." The underdense region is the interconnecting opening between Voids. It is the gaping "hole" in the structural wall.

The explanation applies to any such cold spots observed. When the line of sight is aligned across opposite parallel faces (and especially when extending through several Voids), because of the sparsity of galaxies, the view will be one of a dark region. It would be like looking into a colossal multi-chambered hole in the cosmos.

Extraordinary sequences of galaxy clusters. The evidence under this category is rarely discussed by astronomers and theorists. What it reveals is cosmic structure that is far too systematic-much too orderly to be explained by any $20^{\text {th }}$-century model. The evidence consists of the cluster-void sequences shown schematically in Figure 11 and Figure 12.

Periodic galaxy clusters. The Abell- 85 sequence, which also includes the background clusters Abell 87 and Abell 89, are undoubtedly the most unusual arrangement of galaxy clusters ever observed. The near regular spatial periodicity of the clusters is completely inexplicable with any other theory or hypothesis. No other universe model can explain a cluster-and-void sequence extending for over 10 repetitions! [24] [25]

Another example of this kind of cluster periodicity is the structure known as DC1842-63 with three periodic clusters. A published histogram shows them located at $4500 \mathrm{~km} / \mathrm{s} ; 10,500 \mathrm{~km} / \mathrm{s}$; and about $16,000 \mathrm{~km} / \mathrm{s}$; evidently evenly spaced [26]. (It could be that DC1842-63 is also known as Abell S0301.)

Periodic linear clusters. A completely different sequence arises when the line of sight runs through opposite Minor nodes of the dodecahedral tessellation (Figure 12). The order in this case is as follows: void, triple cluster, void, triple cluster, and so on. Each triple cluster falls along the same axis and consists, first, of a Minor cluster, then a filamentary cluster, a Major cluster, another filamentary cluster, and ends with another Minor cluster. The axis then passes across another Void. The familiar "CFA stick man" is an example of such a void-andtriple-cluster sequence (Figure 9). The near void is part of what is called the Northern Local Supervoid ${ }^{3}$ and the Major node is AGC1656 (aka Coma cluster).

${ }^{3}$ The Northern Local Supervoid is the great underdense region between the "nearby" Virgo cluster and the Coma and Hercules superclusters (both of which are part of the CfA2 Great Wall). 


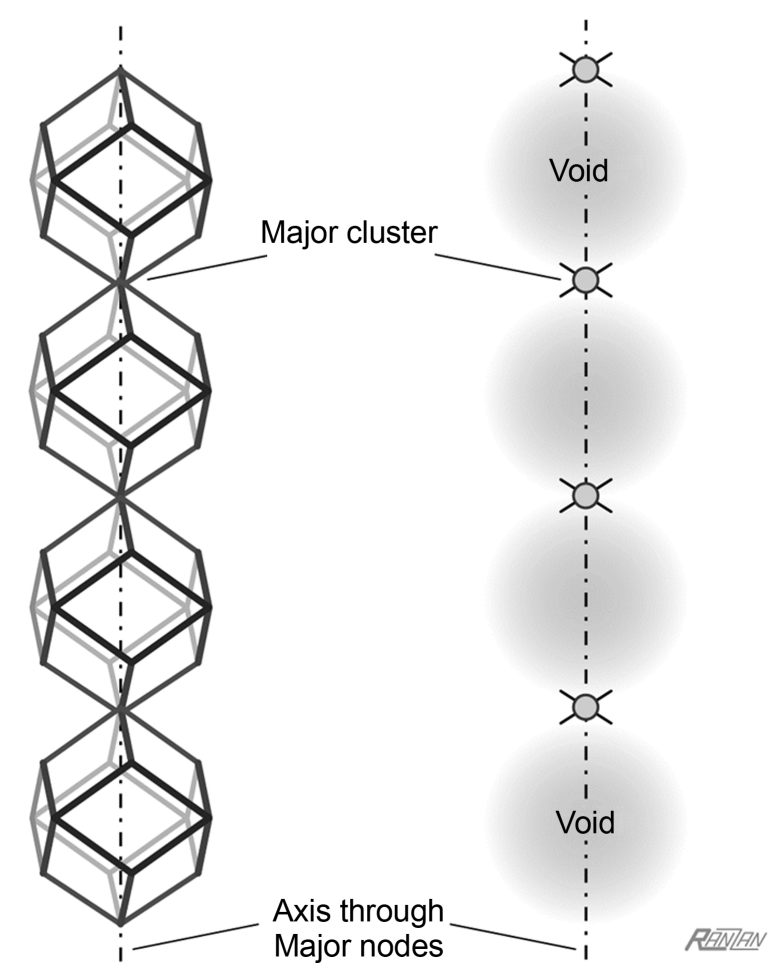

Figure 11. Characteristic pattern found in a close-packing of dodecahedral cosmic cells. The line of sight through opposite Major nodes, as shown here schematically, encounters an alternating sequence of Major galaxy clusters and great Voids. (The surrounding units of the packing have been omitted, for the sake of clarity.) The evidence for this kind of pattern can be found in the Abell-85 system of galaxy clusters.

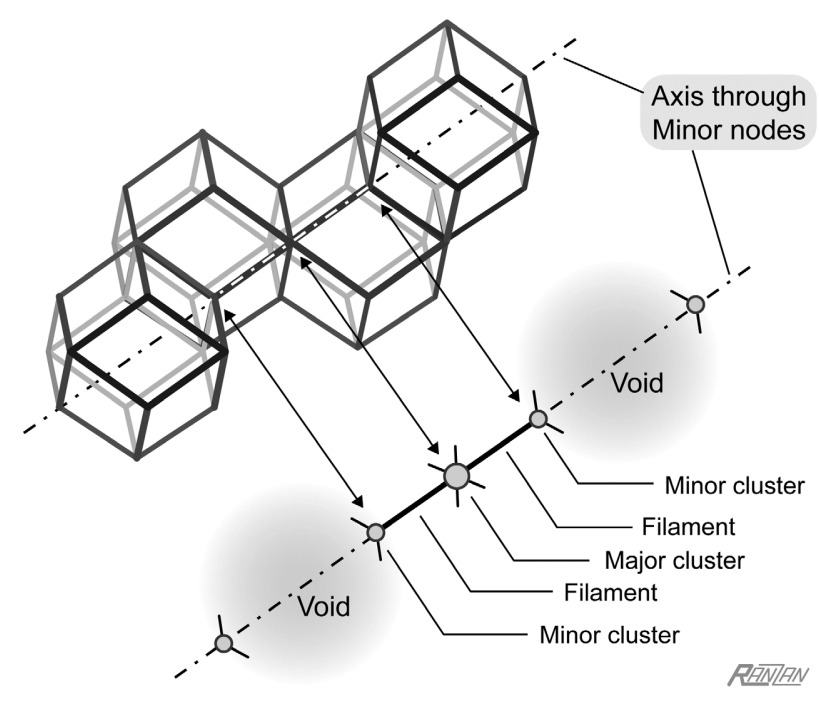

Figure 12. Characteristic pattern found along the line of sight through opposite Minor nodes of dodecahedral cosmic cells. The sequence along an idealized extended axis through Minor nodes always has Voids separated by triple clusters. In other words, the sequence alternates between Voids and triple-chain clusters. (Again, the surrounding cells of the close-packing have been omitted, to better reveal the pattern. In the perspective view, the end units are closer than the middle pair.) See Figure 9 for evidence of this kind of pattern; the Major node there is occupied by the Coma cluster. 
As for the far Void, it has not been identified; it does not seem to have been given an official name.

Again, evidence agrees with prediction.

\section{Summary Items}

\subsection{Cosmic Cells Do Not Expand}

The reason cosmic cells do not expand deserves emphasis. Since this is often misunderstood, here is a recap of what is probably the most important aspect of the cosmic structural cells. Given that the cell interiors, the Voids, are filled with expanding aether, what prevents Voids from growing larger in size? The reason is simply that mass absorbs the aether. (This is also true of energy particles and energy fields.) The existence of matter is sustained by the absorption-consumption of this essence fluid. The material surrounding each Void acts as the sink for the expanding aether-thereby limiting the overall expansion and helping to maintain each cosmic cell as a steady-state system.

"Expansion" was adopted as the core assumption—but it was expansion without extrapolation. The quantitative expansion/growth of the universal medium is balanced by an equal quantity of contraction/consumption/self-vanishment. In other words, there is NO NET expansion on the largest scale! (And this, as it turns out, was one of the overlooked laws of cosmic-scale physics.)

\subsection{Overview}

Remarkably, we started with only that one empirical proposition. (Not even the cosmological principle was adopted as an assumption; and for good reason. Both theory and evidence point to a universe lacking homogeneity and isotropy.) Everything else was based on good evidence and on three important laws of physics that $20^{\text {th }}$-century scientists overlooked-namely those detailed in Parts 1,2 , and 4 in this series of articles:

- "Part 1: The velocity differential propagation of light," which presents the definitive cause of the cosmic redshift [3].

- "Part 2: Energy Generation via Velocity Differential Blueshift." It details Nature's fundamental energy amplification process [5].

- "Part 4: Mass Extinction by Aether Deprivation." It gives the remarkable explanation of how Nature annihilates mass. No ordinary destruction process, this is annihilation in the irreversible terminal sense [7].

Something else those scientists overlooked was a deep and fundamental contrast between individual bodies and collectives of bodies. Although they understood the mutability of stellar and planetary bodies, they utterly failed to recognize the eternal order of the distribution patterns. They never did discover the natural and perpetual order of cosmic-scale structure.

Also, there was something at a truly fundamental level. Although they understood the importance of the space medium, the vacuum, they failed to grasp its ontological nature. 


\subsection{The Sine Qua Non Component}

Undoubtedly, the most important component of the Universe is the omnipresent space medium. Purposely called "aether" herein, as a way of acknowledging the contribution of the Ancient Greeks; and because Albert Einstein emphatically stated that aether exists. But he also made it quite clear that it was not a material aether; it was not like the $19^{\text {th }}$-century aether [27]. Einstein was correct on both counts; unfortunately, the nonmaterial-medium concept was never exploited. When Physics rightfully rejected the material aether, it wrongfully also discarded the whole aether concept. The result was an entire century of obfuscation of the unique nature of the space medium. In any case, it helps to be clear on meanings and avoid any misunderstanding. Here are three relevant definitions of a much-discredited term.

1) The original ether. In Aristotelian physics, the fifth element, the quintessence, of which the "heavens" are made. In Classical physics, it is the invisible medium that diffuses all space.

2) The historic aether: The material medium that fills the apparent emptiness of the universe. Invented by René Descartes and by Isaac Newton; reinvented by many others, including James Clerk Maxwell who used it for his electromagnetic theory; but was discredited and discarded by the young Einstein.

3) The DSSU aether: The subquantum medium that permeates all space. It is the nonmaterial essence of the Universe; it consists of discrete units-fundamental essence oscillators. As a basic space medium, it serves as the propagator of electromagnetic waves. As a space-permeating dynamic medium, it manifests gravitation; its nature is responsible for the several guises of gravity without invoking any requisite force-carriers.

Aether was actually detected and verified in at least six separate experiments during the $20^{\text {th }}$ century (in addition to the Michelson and Morley detection in 1887). But since there was no supporting theory, the findings were either ignored or relegated to the obscurity of footnotes. It was baffling. How could a supposedly nonmaterial medium be detectable? But since the space medium proved to be detectable, then it had to be material! Theorists were trapped in a contradiction. Physical experiments said the space medium is material; while theory said it is nonmaterial. They were trapped by a failure to understand the profound connection between the material and nonmaterial realms of existence. That connection, long-overlooked, is a subquantum aether.

The essential nature of the space medium is that it is nonphysical-nonphysical because it exists at the subquantum level. Furthermore it is mechanical-it consists of discrete entities. See Figure 13.

DSSU aether is the essential component for sustaining all things and all structures, and, in the context of the current article, for the patterns of cosmic structure.

Consider this analogy, borrowed from physicist Evan Harris Walker, on the need for a special kind of mechanics. Just as the electromagnetic forces govern 


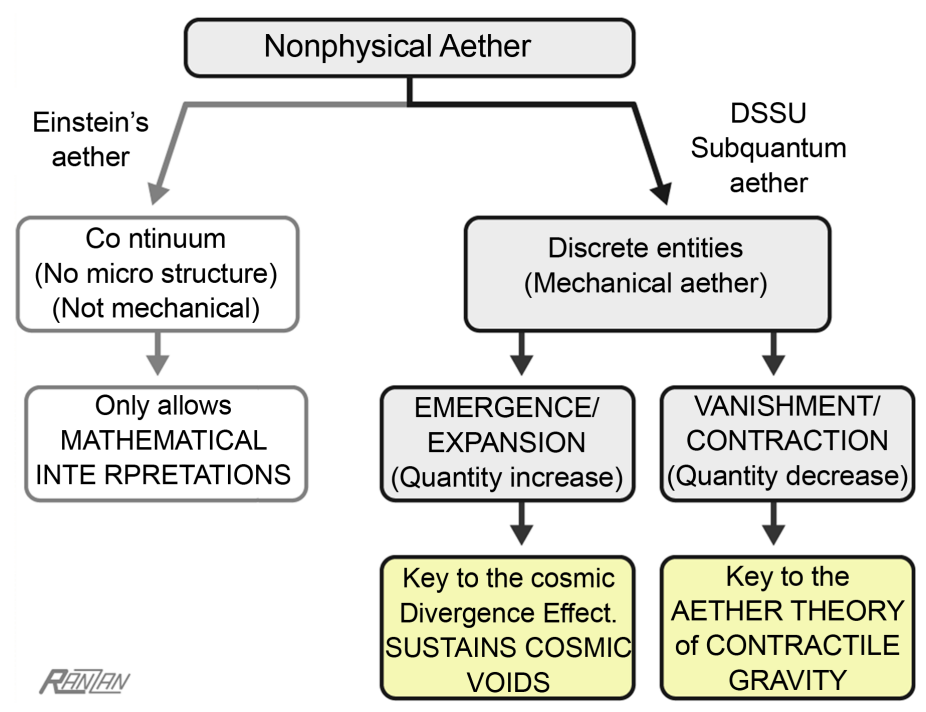

Figure 13. Two theories of nonphysical aether. Einstein's version has no micro structure, hence is not mechanical. DSSU aether consists of discrete units, hence it IS mechanical. Moreover, existing as they do at the subquantum level, these units possess no mass and no energy. Therefore and profoundly, the key mechanical processes of EMERGENCE and VANISHMENT cannot represent a violation of the conventional thermodynamic laws. Thus, a nonmaterial medium underpins cosmic structure.

the structure of atoms and molecules, the gravitation effects dominate the structure of the large-scale objects and the structure of the entire universe. And in order to explain those gravity effects and to understand how the universe as a whole works, a special kind of mechanics is needed. For $20^{\text {th }}$-century scientists, "That special mechanics is known as the general theory of relativity." [28] That, of course, was Einstein's geometric/mathematical model of gravity. (Remember, Einstein acknowledged aether's existence but did not explicitly employ it.) Although of limited applicability in local reference frames, the theory was, and is, incomplete (by Einstein's own admission), and is quite useless (makes wrong predictions) when applied to the greater universe.

The special kind of mechanics essential to understanding the Universe is $d y$ namic-aether gravity — the gravity theory based on the above mechanical aether (Figure 13). A key component of the laws of physics, one that was overlooked by $20^{\text {th }}$-century scientists, was the mechanics of a nonmaterial-and-dynamic aether.

Again, there is nothing more fundamental, or so fundamentally important, than DSSU-defined aether.

\section{Conclusions}

The great discovery of the last century was that the Universe is cellular. During its many decades, astronomers succeeded in showing some amazing regularities. Meanwhile, the theoretical experts struggled to come up with plausible explanations using theories of the universe that had failed long ago and should have been abandoned. Yet the challenge was straightforward. It was not a matter of "if" but of "how." The question was, In what way is the universe cellular?... And 
the answer can be had by a strategy of elimination. Just examine all the possible suspects that may be behind the "anomalous" patterns, the various configurations of galaxies described earlier. The list of candidates is quite short:

- First, consider what the old theories predicted: Random chaotic spherical cells, random in size and chaotic in motion. But randomness was just the opposite of the orderly structure evident in the real-world.

- What about cube shaped cosmic cells? Too simplistic and, in a gravity-ruled world, highly unstable.

- What about truncated-octahedra? This was examined by "The Cosmic Web" expert Richard Gott ([12], p. 107). It is true that truncated-octahedra do close pack in a space-filling arrangement. However, this polyhedron has two obvious problems-it lacks right-angled walls and its nodes are all the same. With all nodes being identical, it becomes difficult to explain galaxy-cluster-size variation.

- Lastly, there is the rhombic dodecahedron-a shape for which all the pieces of evidence fit. There simply are no other cell types-no other symmetrical or semi-symmetrical space dividing cell shapes.

There is only one cell shape, only one tessellation pattern, that can explain all three key structural features described earlier and illustrated in Figure 8, Figure 11, and Figure 12-only the rhombic dodecahedral configuration will work.

But replacing randomness with systematic cellularity comes with consequences. The body of the evidence and its interpretation within the DSSU framework leads to an unavoidable reassessment of a long-cherished belief. The implication is that our Universe is not entirely subject to the cosmological principle. A universe inherently cellular is structurally somewhat like a liquid crystal. Both have symmetry properties; both are nonisotropic. The Universe may well be a uniform system of cells; and be described as a homogeneous "packing" of those cells; but it cannot be described as being isotropic.

The revolutionary difference. There is a revolutionary difference of interpretation of the observational evidence of the void-cluster network. Under the $20^{\text {th }}$-century view, cosmic cellular structure is merely a phenomenological condition, a statistical fluke, while under the $21^{\text {st }}$-century view-DSSU world view-it is an inherent state.

A triumph of reason. As the DSSU foundational postulate and the overlooked laws of physics (previously identified) were pieced together and applied to the Universe, a point was reached where one could finally grasp the nature of cosmic structure-its underlying patterns and its steady state aspects. Theory-based predictions were matched up with astronomical evidence. Theory, predictions, and observations, all were found to fit smoothly together into a comprehensive vision of reality.

Without philosophical absurdities (universe genesis); without unscientific extrapolations (exploding cosmos); without outright paradoxes (singularity black holes); without physical-law breakdown (extreme gravitational collapse); and without fantasy matter (invisible dark matter), the Dynamic Steady State Universe stands 
sui generis as the problem-free cosmology.

A closing thought:

When the wise men of their time, long ago, assured the masses that the heavens, the celestial sphere, had a rotational mobility they were wrong. When the wise men of the $20^{\text {th }}$ century assured the masses that the heavens have a radial mobility-an accelerating outward-bound mobility—they too were wrong.

$$
* * *
$$

\section{Conflicts of Interest}

The author declares no conflicts of interest.

\section{References}

[1] Susskind, L. (2006) The Cosmic Landscape, String Theory and the Illusion of Intelligent Design. Back Bay Books, Little, Brown and Co., New York, 309.

[2] Ranzan, C. (2016) The Nature of Gravitational Collapse. American Journal of Astronomy and Astrophysics, 4, 15-33. https://doi.org/10.4006/0836-1398-33.2.163

[3] Ranzan, C. (2020) Law of Physics 20th-Century Scientists Overlooked (Part 1): The Velocity Differential Propagation of Light. Physics Essays, 33, 163-174. https://doi.org/10.4006/0836-1398-33.2.163

[4] Ranzan, C. (2014) Cosmic Redshift in the Nonexpanding Cellular Universe: Velocity-Differential Theory of Cosmic Redshift. American Journal of Astronomy \& Astrophysics (AJAA), 2, 47-60. https://doi.org/10.11648/j.ajaa.20140205.11

[5] Ranzan, C. (2020) Law of Physics 20th-Century Scientists Overlooked (Part 2): Energy Generation via Velocity Differential Blueshift. Physics Essays, 33, 289-298. https://doi.org/10.4006/0836-1398-33.3.289

[6] Ranzan, C. (2018) Natural Mechanism for the Generation and Emission of Extreme Energy Particles. Physics Essays, 31, 358-376.

https://doi.org/10.4006/0836-1398-31.3.358

[7] Ranzan, C. (2021) Law of Physics 20th-Century Scientists Overlooked (Part 4): Mass Extinction by Aether Deprivation. Journal of High Energy Physics, Gravitation and Cosmology, 7, 191-209. https://doi.org/10.4236/jhepgc.2021.71010

[8] Ranzan, C. (2018) The Nature of Gravity-How One Factor Unifies Gravity's Convergent, Divergent, Vortex, and Wave Effects. International Journal of Astrophysics and Space Science, 6, 73-92. https://doi.org/10.11648/j.ijass.20180605.11

[9] Halzen, F. (2015, October) Neutrinos at the Ends of the Earth. Scientific American, 61. https://doi.org/10.1038/scientificamerican1015-58

[10] Pike, O.J., Mackenroth, F., Hill, E.G. and Rose, S.J. (2014) A Photon-Photon Collider in a Vacuum Hohlraum. Nature Photonics, 8, 434-436.

https://doi.org/10.1038/nphoton.2014.95

[11] Ranzan, C. (2016) Large-Scale Cell Structure of the Dynamic Steady State Universe. American Journal of Astronomy \& Astrophysics, 4, 65-77. https://doi.org/10.11648/j.ajaa.20160406.11

[12] Gott, J. R. (2016) The Cosmic Web, Mysterious Architecture of the Universe. Princeton University Press, Princeton. https://doi.org/10.1515/9781400873289

[13] Ota, N. and Mitsuda, K. (2002) X-Ray Study of Seventy-Nine Distant Clusters of Galaxies: Discovery of Two Classes of Cluster Size. The Astrophysical Journal, 567, L23-L26. https://doi.org/10.1086/339852 
[14] Abell, G.O. (1982) Exploration of the Universe. 4th Edition, Saunders College Publishing, New York, 613.

[15] Kraan-Korteweg, R.C. (2000) Galaxies behind the Milky Way and the Great Attractor. In: Page, D. and Hirsch, J.G., Eds., From the Sun to the Great Attractor, Vol. 556, Springer, Berlin, 301-344. https://doi.org/10.1007/3-540-45371-7 8 http://arxiv.org/abs/astro-ph/0006199

[16] Broadhurst, R., Ellis, R.S., Koo, D.C. and Szalay, A.S. (1990) Large-Scale Distribution of Galaxies at the Galactic Poles. Nature, 343, 726-728. https://doi.org/10.1038/343726a0

[17] Kurki-Suonio, H., Mathews, G.J. and Fuller, G.M. (1990) Deviation from Periodicity in the Large-Scale Distribution of Galaxies. Astrophysical Journal, 356, L5. https://doi.org/10.1086/185738

[18] Mahan, B.H. (1969) University Chemistry. 2nd Edition, Addison-Wesley Publishing Co., Boston, 109-110.

[19] Ebbing, D.D. and Gammon, S.D. (1999) General Chemistry. 6th Edition, Houghton Mifflin Co., New York, 476-477.

[20] Fairall, A.P., Paverd, W.R. and Ashley, R.P. (1994) Visualization of Nearby LargeScale Structures. ASP Conference Series, 67, 21-30. http://www.adsabs.harvard.edu/full/1994ASPC...67...21F

[21] Fairall, A.P., Turner, D., Pretorius, M.L., Wiehahn, M., McBride, V., Vaux, G. de and Woudt, P.A. (2005) Percolation Properties of Nearby Large-Scale Structures: Every Galaxy Has a Neighbour. ASP Conference Series, 329, 229. arXiv:astro-ph/0411437. https://ui.adsabs.harvard.edu/abs/2005ASPC..329..229F

[22] Fairall, A.P., Palumbo, G.G.C., Vettolani, G., Kauffmann, G., Jones, A. and Baiesi-Pillastrini, G. (1990) Large-Scale Structure in the Universe: Plots from the Updated Catalogue of Radial Velocities of Galaxies and the Southern Redshift Catalogue. Monthly Notices of the Royal Astronomical Society, 247, 21-25.

[23] Fairall, A.P. (1995) Large-Scale Structures in the Distribution of Galaxies. Astrophysics and Space Science, 230, 229-234. https://doi.org/10.1007/BF00658183 https://ui.adsabs.harvard.edu/abs/1995Ap\&SS.230..225F

[24] Ranzan, C. (2015) DSSU Validated by Redshift Theory and Structural Evidence. Physics Essays, 28, 455-473. https://doi.org/10.4006/0836-1398-28.4.455

[25] Durret, F., Felenbok, P., Gerbal, D., Guibert, J., Lobo, C. and Slezak, E. (1996) Redshift and Photometric Survey of the X-Ray Cluster of Galaxies Abell 85. The Messenger, 84, 20-23. http://adsabs.harvard.edu/abs/1996Msngr..84...20D

[26] Malumuth, E.M., Kriss, G.A., Dixon, W.V.D., Ferguson, H.C. and Ritchie, C. (1992) Dynamics of Clusters of Galaxies with Central Dominant Galaxies. Astronomical Journal, 104, 495-530. https://doi.org/10.1086/116250 http://articles.adsabs.harvard.edu//full/1992AJ....104..495M

[27] Einstein, A. (1922) Sidelights on Relativity, Ether and the Theory of Relativity. Jeffery, G.B. and Perret, W., Eds., Methuen \& Co., London. http://www.gutenberg.org/ebooks/7333

[28] Walker, E.H. (2000) The Physics of Consciousness. Perseus Books, Cambridge, 313. 